$$
g(0)=0 \text {. }
$$

By the theorem on the ordinary differential inequality it follows from (18), (19) that

$$
g(x) \leqslant 0 \quad \text { for } \quad 0 \leqslant x \leqslant 1 .
$$

By (8), (7), (4) it follows that $g(x)=0$. Hence by (8)

$$
u_{i} \equiv 0 \quad(i=1, \ldots, m) \quad \text { for } \quad \begin{array}{r}
0<x<1, \quad\left|y_{k}\right| \leqslant 1 \\
(k=1, \ldots, n) \quad \text { q. e. d. }
\end{array}
$$

Remark. Applying Hadamard's lemma we may obtain an analogue of theorem 2 from [1] for the mixed problem for the following nonlinear system of partial differential equations of the second order:

$$
\begin{array}{r}
\frac{\partial u_{i}}{\partial x}=F_{i}\left(Z, U, \frac{\partial U}{\partial y_{1}}, \ldots, \frac{\partial U}{\partial y_{n}}, \frac{\partial^{2} U}{\partial y_{1} \partial y_{1}}, \ldots, \frac{\partial^{2} U}{\partial y_{1} \partial y_{n}}, \frac{\partial^{2} U}{\partial y_{2} \partial y_{1}}, \ldots, \frac{\partial^{2} U}{\partial y_{n} \partial y_{n}}\right) \\
\left(i=1, \ldots, m ; U=\left(u_{1}, \ldots, u_{m}\right)\right) .
\end{array}
$$

\title{
References
}

[1] A. Plis, On the uniqueness of the non-negative solution of the homogeneous Oauchy problem for a system of partial differential equations, Ann. Polon. Math. 2 (1955), p. $314-318$.

[2] - The problem of uniqueness for the solution of a system of partial differen. tial equations, Bull. Acad. Polon: Sci., Cl. III, 2 (1954), p. 55-57.

\author{
Regu par la Rédaction le 15. 12. 1958
}

\section{POLONICI MATHEMATICI}

VII (1960)

\section{Sur la stabilité asymptotique des solutions d'un système d'équations différentielles}

par Z. OPIAL (Kraków)

1. Considérons le système de deux équations différentielles

$$
X^{\prime}=f(X, t)
$$

où $X$ désigne le vecteur $\left(x_{1}, x_{2}\right)$ et $f(X, t)$ est une fonction vectorielle $\left(f_{1}\left(x_{1}, x_{2}, t\right), f_{2}\left(x_{1}, x_{2}, t\right)\right)$ dont les composantes sont continues par rapport à $\left(x_{1}, x_{2}, t\right)$ et de classe $C^{1}$ par rapport à $\left(x_{1}, x_{2}\right)$ dans tout l'espace à trois dimensions $\left(x_{1}, x_{2}, t\right)$.

Désignons par $X\left(t ; X_{0}, t_{0}\right)$ la solution (unique en vertu des hypothèses précédentes) du système (1) qui passe par le point $\left(X_{0}, t_{0}\right)$.

Soit $K$ un ensemble fermé, borné et simplement connexe du plan $\left(x_{1}, x_{2}\right)$. On dit (cf. [8], p. 83) que le système (1) est relativement borné dans l'ensemble $K$ si pour tout $X_{0} \epsilon K$ et tout $t_{0}$ on a $X\left(t ; X_{0}, t_{0}\right) \epsilon K$ pour $t \geqslant t_{0}$.

On dit qu'une solution $X(t) d u$ système (1) est asymptotiquement stable dans l'ensemble $K$ si le système considéré est relativement borné dans cet ensemble et si pour tout $X_{0} \in K$ et tout $t_{0}$ on a la relation

$$
\lim _{h \rightarrow+\infty}\left|X\left(t ; X_{0}, t_{0}\right)-X(t)\right|=0,
$$

où $|X|$ désigne la longueur euclidienne du vecteur $X$.

De la relation (2) il vient que l'on a quels que soient les points $X_{1}$, $X_{2} \in K$ et $t_{0}$,

$$
\lim _{t \rightarrow+\infty}\left|X\left(t ; X_{1}, t_{0}\right)-X\left(t ; X_{2}, t_{0}\right)\right|=0
$$

ce qui signifie que toute solution du système (1) passant par un point de l'ensemble $K$ est forcément asymptotiquement stable dans cet ensemble. Donc, au lieu de dire qu'une solution choisie du système envisagé est asymptotiquement stable dans $K$ on peut dire tout simplement que c'est le système (1) lui-même qui est asymptotiquement stable dans cet ensemble. 
2. Récemment G. Seifert [8] a établi un critère général de stabilité asymptotique du système (1) et il l'a appliqué à la démonstration de la stabilité des solutions périodiques d'un système du type (1) à second membre périodique par rapport à la variable indépendante $t$. Dans la présente note je me propose de montrer comment on peut obtenir, par une simple modification de la méthode de G. Seifert, une méthode encore plus maniable et quelquefois plus efficace. Cela nous permettra de simplifier considérablement la démonstration d'un critère de stabilité de la solution périodique de l'équation différentielle du second ordre

$$
x^{\prime \prime}+f(x) x^{\prime}+g(x)=p(t),
$$

établi par G. Seifert dans la note mentionnée (cf. [8], p. 86, Theorem 2).

Nous nous bornons à envisager un système de deux équations, mais de pareilles considérations peuvent aussi s'appliquer - comme il ost facile de- le voir - anx systèmes plus généraux contenant un nombre arbitraire d'équations.

Notons enfin qu'une méthode analogue à celle exposée dans le présent travail peut être aussi appliquée à la comparaison non seulement de deux solutions d'un système donné, mais aussi à celle des solutions de systèmes différents (voir p. ex. Z. Opial [4] ot [5]).

3. Soit $K$ un ensemble plan fermé, borné et simplement connexe dont la frontière est une courbe composée d'un nombre fini d'ares simples de classe $C^{1}$ (nous supposons de plus qu'en chaque point de cette frontière il existe des demi-tangentes - nous dirons dans toute la suite qu'une telle courbe est de classe $\tilde{C}^{1}$ ). Supposons que le système (1.) soit relativement borné dans cet ensemble et soient $X_{1}, X_{2}$ deux points arbitraires appartenant à $K$. Joignons le point $X_{1}$ an point $X_{2}$ par un arc simple contenu entièrement dans l'ensemble $K$ :

$$
\text { (C) } \quad X=S(u): \quad x_{1}=s_{1}(u), \quad x_{2}=s_{2}(u) \quad(0 \leqslant u \leqslant 1) .
$$

Supposons que les fonctions $s_{i}(u)(i=1,2)$ soient de classe $C^{1}$ dans tout l'intervalle $\langle 0,1\rangle$ et que l'on ait $S(0)=X_{1}$ et $S(1)=X_{2}$. Désignons par $C(t)$ la courbe plane donnée par les équations paramétriques

$(C(t)) \quad X=S(t, u)=X\left(t ; S(u), t_{0}\right): \quad x_{1}=s_{1}(t, u), \quad x_{2}=s_{2}(t, u)$

On a évidemment

$$
\left(t \geqslant t_{0}, 0 \leqslant u \leqslant 1\right. \text { ). }
$$

$$
\left|X\left(t ; X_{1}, t_{0}\right)-X\left(t ; X_{2}, t_{0}\right)\right|=|S(t, 0)-S(t, 1)| \leqslant L(t),
$$

où $L(t)$ désigne la longueur de l'arc $C(t)$, c'est-à-dire

$$
L(t)=\int_{0}^{1}\left\{\left(\frac{\partial s_{1}(t, u)}{\partial u}\right)^{2}+\left(\frac{\partial s_{2}(t, u)}{\partial u}\right)^{2}\right\}^{1 / 2} d u .
$$

Il est facile de vérifier que les fonctions

$$
v_{1}\left(t_{0} u\right)=\partial s_{1}(t, u) / \partial u, \quad v_{2}(t, u)=\partial s_{2}(t, u) / \partial u
$$

satisfont pour $t \geqslant t_{0}$ et $0 \leqslant u \leqslant 1$ au système d'équations différentielles linéaires

$$
\frac{d v_{i}}{d t}=\sum_{i, j=1}^{2} f_{i j}\left(s_{1}(t, u), s_{2}(t, u), t\right) v_{j} \quad(i=1,2),
$$

où $f_{i j}\left(x_{1}, x_{2}, t\right)=\partial f_{i}\left(x_{1}, x_{2}, t\right) / \partial x_{j}$.

Si les fonctions $v_{1}(t, u), v_{2}(t, u)\left(t \geqslant t_{0}, 0 \leqslant u \leqslant 1\right)$ sont bornées dans leur ensemble dans l'intervalle $\left\langle t_{0},+\infty\right)$ et si, de plus, elles tendent vers zéro lorsque $t$ tend vers l'infini, alors la fonction $L(t)$ tend vers zéro et, par conséquent, de l'inégalité (5) on tire la relation (3).

4. Définimion. Nous dirons que le système (1) jouit de la propriété (M) dans l'ensemble $K$ si pour tout $X_{0} \in K$, tout $t_{0}$ et tout nombre positif $A$ il existe un nombre $B>0$ tel que toute solution $\left(v_{1}(t), v_{2}(t)\right)$ du système d'équations différentielles linéaires

$$
\frac{d v_{i}}{d t}=\sum_{i, j=1}^{2} f_{i j}\left(X\left(t ; X_{0}, t_{0}\right), t\right) v_{j} \quad(i=1,2)
$$

aux valeurs initiales bornées par $A$ pour $t=t_{0}$ :

$$
v_{1}^{2}\left(t_{0}\right)+v_{2}^{2}\left(t_{0}\right) \leqslant A \text {, }
$$

satisfait dans tout l'intervalle $\left\langle t_{0},+\infty\right)$ à l'inégalité

$$
v_{1}^{2}(t)+v_{2}^{2}(t) \leqslant B
$$

et tend vers zéro lorsque $t$ croît indéfiniment:

$$
\lim _{t \rightarrow+\infty} v_{1}(t)=\lim _{t \rightarrow+\infty} v_{2}(t)=0
$$

Pour toute solution arbitraire fixe $X\left(t ; X_{0}, t_{0}\right)$ du système (1) le système $(6)$ est linéaire et homogène. Donc, il suffit d'envisager deux solutions linéairement indépendantes de ce système. Si elles vérifient les relations (9), il en est de même de toute autre solution du système (6) et pour tout $A>0$ (et $t_{0}$ fixe) il existe un $B$ tel que l'inégalité (7) entraîne l'inégalité (8). Et inversement, pour tout $B>0$ il existe un $A>0$ pour lequel (7) entraîne (8). On dit dans ce cas que la solution identiquement nulle du système (6) est asymptotiquement stable au sens de Liapounoff (ef. I. G. Malkine [3], p. 12). Or, la constante $B$ dépend non seulement de la valeur de $A$, mais - en général - aussi de la solution choisie $X\left(t ; X_{0}, t_{0}\right)$ du système (1) (c'est-à-dire du point $\left(X_{0}, t_{0}\right)$ ). 
On peut donc dire que le système (1) jouit de la propriété (M) dans l'ensemble $K$ si la solution identiquement nulle des systèmes (6) est asymptotiquement stable uniformément par rapport à $X_{0} \in K$ et $t_{0}$ arbitraire.

5. En nous appuyant sur ce que nous arons dit au $n^{0} 3$ nous pouvons énoncer le théorème suivant:

THÉORÈme 1. Soit $K$ un ensemble plan fermé, borné et simplement connexe, limité par une courbe de classe $\tilde{O}^{1}$. Si le système (1) est relativement borné dans $K$ et $y$ jouit de la propriété $(\mathrm{M})$, le système (1) est asymptotiquement stable dans cet ensemble.

En effet, deux points arbitraires $X_{1}$ et $X_{2}$ de l'ensemble $K$ peuvent être joints par une courbe $C$ de classe $C^{1}$ contenue entièrement dans $K$. Si le système (1) jouit de la propriété $(\mathbf{M})$, la longueur $L(t)$ de la courbe $O(t)$ (cf. $\mathrm{n}^{0} 3$ ) tend vers zéro lorsque $t$ croît indéfiniment. On a done la relation (3) et le théorème 1 se trouve ainsi démontré.

On peut de même démontrer que pour tout $t_{0}$ fixe la convergence exprimée par la formule (3) est uniforme par rapport aux points $X_{1}$ et $X_{2}$ variant dans l'ensemble $K$. Il suffit à cet effet de prendre pour $C$ la courbe qui constitue la frontière de l'ensemble $K$. La longueur de la courbe correspondante $C(t)\left(t \geqslant t_{0}\right)$ tend vers zéro lorsque $t$ tend vers l'infini, ce qui signifie que la convergence envisagée est bien uniforme.

Dans le cas où, lorsque $A$ tend vers zéro, $B$ tend aussi vers zéro, toute solution $X\left(t ; X_{0}, t_{0}\right)$ du système (1) ( $X_{0}$ appartient à l'intérieur de l'ensemble $K$ et $t_{0}$ est arbitraire) est asymptotiquement stable au sens de Liapounoff. En effet, dans cette hypothèse à tout $\varepsilon>0$ on peut faire correspondre un $\delta>0$ tel que

$1^{\circ}$ le voisinage $V:\left|X-X_{0}\right| \leqslant \delta$ appartienne à $K$;

$2^{\circ}$ en prenant pour courbe $O$ joignant les points $X_{0}$ et $X_{1} \in V$ le segment $\bar{X}_{0} \bar{X}_{1}$, la longueur de la courbe correspondante $C(t)\left(t \geqslant t_{0}\right)$ soit au plus égale à $\varepsilon$.

6. Soit $\Omega\left(X ; v_{1}, v_{2}\right)=\Omega\left(x_{1}, x_{2} ; v_{1}, v_{2}\right)$ une forme quadratique des variables $v_{1}, v_{2}$ dont les coefficients sont des fonctions de classe $O^{1}$ des variables $x_{1}, x_{2}$ :

$$
\Omega\left(x_{1}, x_{2} ; v_{1}, v_{2}\right)=\sum_{i, j=1}^{2} g_{i j}\left(x_{1}, x_{2}\right) v_{i} v_{j} .
$$

Soit $v_{1}=v_{1}(t), v_{2}=v_{2}(t)$ une solution arbitraire du système (6). On a alors

$$
\text { (10) } \begin{aligned}
\frac{d}{d t} \Omega\left(x_{1}\left(t ; X_{0}, t_{0}\right)\right. & \left., x_{2}\left(t ; X_{0}, t_{0}\right) ; v_{1}(t), v_{2}(t)\right) \\
& =-\Omega_{1}\left(x_{1}\left(t ; X_{0}, t_{0}\right), x_{2}\left(t ; X_{0}, t_{0}\right), t ; v_{1}(t), v_{2}(t)\right)
\end{aligned}
$$

où $\Omega_{1}\left(x_{1}, x_{2}, t ; v_{1}, v_{2}\right)$ est une forme quadratique des variables $v_{1}, v_{2}$ à coefficients dépendant des variables $x_{1}, x_{2}$ et $t$.

Supposons que la forme $\Omega$ soit définie positive pour tout point $\left(x_{1}, x_{2}\right)$ de l'ensemble $K$. L'ensemble $K$ étant compact, il existe deux nombres positifs $\alpha$ et $\beta$ tels que l'on a pour tout point $\left(x_{1}, x_{2}\right)$ de cet ensemble et tout couple de nombres $v_{1}$ et $v_{2}$ :

$$
\alpha\left(v_{1}^{2}+v_{2}^{2}\right) \leqslant \Omega\left(x_{1}, x_{2} ; v_{1}, v_{2}\right) \leqslant \beta\left(v_{1}^{2}+v_{2}^{2}\right) .
$$

Admettons de plus que la forme quadratique $\Omega_{1}$ soit définie positive dans l'ensemble $K$, uniformément par rapport à $t$, c'est-à-dire qu'il existe un nombre positif $\gamma$ tel que l'on ait

$$
\gamma\left(v_{1}^{2}+v_{2}^{2}\right) \leqslant \Omega_{1}\left(x_{1}, x_{2}, t ; v_{1}, v_{2}\right)
$$

pour tout point $\left(x_{1}, x_{2}\right)$ de l'ensemble $K$ et $v_{1}, v_{2}, t$ arbitraires.

Ceci étant admis, des relations (10)-(12) on obtient l'inégalité différentielle

$$
\frac{d}{d t} \Omega\left(X\left(t ; X_{0}, t_{0}\right) ; v_{1}(t), v_{2}(t)\right) \leqslant-\frac{\gamma}{\beta} \Omega\left(X\left(t ; X_{0}, t_{0}\right) ; v_{1}(t), v_{2}(t)\right) .
$$

Il en résulte que pour tout $t \geqslant t_{0}$ on a l'inégalité

$$
\Omega\left(X\left(t: X_{0}, t_{0}\right) ; v_{1}(t), v_{2}(t)\right) \leqslant \Omega\left(X\left(t_{0} ; X_{0}, t_{0}\right) ; v_{1}\left(t_{0}\right), v_{2}\left(t_{0}\right)\right)
$$

et de plus

$$
\lim _{h \rightarrow+\infty} \Omega\left(X\left(t ; X_{0}, t_{0}\right) ; v_{1}(t), v_{2}(t)\right)=0 .
$$

En vertu de la première des inégalités (11) il en vient

$$
\lim _{t \rightarrow+\infty}\left(v_{1}^{2}(t)+v_{2}^{2}(t)\right)=0 .
$$

Cela signifie que, dans nos hypothèses sur les formes quadratiques $\Omega$ et $\Omega_{1}$, le système (1) jouit de la propriété (M) dans l'ensemble $K$. Nout avons ainsi démontré un théorème analogue au théorème de G. Seifert (cf. [8], p. 85, Theorem 1), à savoir:

THÉoR̀̀me 2. Soit $K$ un ensemble plan fermé, borné et simplemens connexe dont la frontière est une courbe fermée de classe $\tilde{\sigma}^{1}$. Si le système (1) est relativement borné dans l'ensemble $K$, s'il existe une forme quadratique $\Omega\left(x_{1}, x_{2} ; v_{1}, v_{2}\right)$ des variables $v_{1}, v_{2}$, ̀̀ coefficients de classe $C^{1}$ par rapport $\dot{a} x_{1}, x_{2}$, satisfaisant aux inégalités (11), et si la forme quadratique $\Omega_{1}\left(x_{1}, x_{2}, t ; v_{1}, v_{2}\right)$ déterminée par la formule (10) vérifie l'inégatité (12), le système (1) est asymptotiquement stable dans l'ensemble $K$. 
Dans le cas envisagé on peut appliquer la remarque finale du $n^{0} 5$. Du théorème précédent on obtient donc immédiatement le corollaire suivant:

Corollatre 1. Dans les hypothèses du théorème 2 toute solution $X\left(t ; X_{0}, t_{0}\right) d u$ système (1), où $X_{0}$ appartient à l'intérieur de l'ensemble $K$ et $t_{0}$ est arbitraire, est asymptotiquement stable au sens de Liapounoff.

7. Considérons maintenant le système

$$
x^{\prime}=y-H^{\prime}(x), \quad y^{\prime}=-g(x)+p(t)
$$

qui s'obtient de l'équation (4) si l'on pose

$$
F(x)=\int_{0}^{x} f(s) d s \quad \text { et } \quad y=x^{\prime}+H^{\prime}(x) .
$$

Pour ce système il est facile de démontrer le théorème suivant (cf. [8], p. 86, Theorem 2 et aussi [5], théorème 4):

THÉORÈmE 3 . Soit $K$ un ensemble plan dont la frontière est une courbe de classe $\tilde{C}^{1}$ et dans lequel le système (13) est relativement borné. Si les fonctions $f(x), g^{\prime \prime}(x)$ et $p(t)$ sont continues et si l'on a, pour tout point $(x, y)$ appartenant à $K$, les inégalités

$$
\left|g^{\prime \prime}(x)\right||y-F(x)|<2 f(x) g^{\prime}(x), \quad f(x)>0,
$$

le système (13) est asymptotiquement stable dans l'ensemble $K$.

Démonstration. Dans le cas envisagé le système (6) admet la forme suivante:

$$
v_{1}^{\prime}=v_{2}-f(x(t)) v_{1}, \quad v_{2}^{\prime}=-g^{\prime}(x(t)) v_{1},
$$

où $(x(t), y(t))$ est une solution du système (13) contenue dans l'ensemble $K$ pour $t \geqslant t_{0}$. Soit $\left(v_{1}(t), v_{2}(t)\right)$ une solution arbitraire du système (15). Posons

$$
H(t)=g^{\prime}(x(t)) v_{1}^{2}(t)+v_{2}^{2}(t) .
$$

En vertu de (13) on a

$$
H^{\prime}(t)=-\left\{2 g^{\prime}(x(t)) f(\infty(t))-g^{\prime \prime}(x(t))[y(t)-F(x(t))]\right\} v_{1}^{2}(t) .
$$

En tenant compte de la première des inégalités (14) on en obtient l'inégalité

$$
H^{\prime}(t) \leqslant-\varepsilon v_{1}^{2}(t) \quad\left(t \geqslant t_{0}\right)
$$

$\varepsilon$ étant une constante positive.
En vertu des inégalités (14) $g^{\prime}(x)>0$ et, par conséquent, la fonction $H(t)$ est non négative. En raison de l'inégalité (16) on a pour tout $t \geqslant t_{0}$ :

$$
H(t)=g^{\prime}(x(t)) v_{1}^{2}(t)+v_{2}^{2}(t) \leqslant H\left(t_{0}\right),
$$

d'où il vient qu'à tout $A>0$ on peut faire correspondre un $B>0$ tel que l'inégalité (7) entraîne (8).

D'autre part, de l'inégalité (16) et du fait que la fonction $H(t)$ est non négative il vient que

$$
\int_{t_{0}}^{\infty} v_{1}^{2}(s) d s<+\infty
$$

ce qui n'est possible que si

$$
\lim _{t \rightarrow+\infty} v_{1}(t)=0
$$

puisque la dérivée $v_{1}^{\prime}(t)$ est bornée en raison de la première des équations (15).

Mais, lorsque $t$ tend vers l'infini, la fonction $H(t)$ tend vers une limite non négative $H(\infty)$. On a donc, tenant compte des équations (15) et de la définition de la fonction $H(t)$ :

$$
\lim _{t \rightarrow+\infty} v_{1}^{\prime}(t)=\lim _{t \rightarrow+\infty} v_{2}(t)=\sqrt{H(\infty)}
$$

d'où, en raison de (17), il vient que l'on doit avoir $H(\infty)=0$. Enfin, de (17) et (18) il résulte que les fonctions $v_{1}(t)$ et $v_{2}(t)$ satisfont aux relations (9).

Nous avons ainsi démontré que le système (13) jouit de la propriété (M) dans l'ensemble $K$ et, par suite, le théorème 3 résulte immédiatement $\mathrm{du}$ théorème 1 .

Remarque. La démonstration du théorème 3 que nous venons d'exposer ici est non seulement plus simple que celle de G. Seifert, mais aussi elle nous permet de rejeter l'hypothèse, faite par lui, que la fonction $f(x)$ est de classe $C^{1}$. Par conséquent, la même hypothèse peut être omise partout où l'on emploie ce théorème (cf. p. ex. G. Seifert [9]).

8. Supposons maintenant que la fonction $p(t)$ soit périodique de période $\omega$. Si les hypothèses du théorème 3 sont vérifiées, il existe dans l'ensemble $K$ une solution périodique du système (13) ayant la même période. De la conclusion de ce théorème il résulte de plus que cette solution périodique est unique et asymptotiquement stable dans l'ensemble $K$. 
9. De même qu'au $\mathrm{n}^{\circ} 6$ on peut appliquer aux considérations $\mathrm{du}$ $\mathrm{n}^{\circ} 7$ la remarque finale $d u \mathrm{n}^{\circ} 5$. On obtient ainsi le corollaire suivant:

CorollaIre 2. Si les hypothèses du théorème 3 sont satisfaites, toute solution $(x(t), y(t))$ du système (13) passant par un point intérieur de l'ensemble $K$ est asymptotiquement stable au sens de Liapounoff.

10. Posons

$$
F^{\prime}(x)=\int_{0}^{x} f(u) d u, \quad G(x)=\int_{0}^{x} g(u) d u, \quad P(t)=\int_{0}^{t} p(u) d u
$$

et supposons que ces fonctions satisfassent aux conditions suivantes:

$$
\begin{aligned}
& \quad f(x)>0 \quad \text { pour tout } x, \lim _{|x| \rightarrow \infty} F(x) \operatorname{sgn} x=+\infty, \\
& \text { (20) } x g(x)>0 \quad \text { pour tout } x \neq 0, \lim _{|x| \rightarrow \infty} G(x)=+\infty,
\end{aligned}
$$

(21) les fonctions $p(t)$ et $P(t)$ sont bornées pour tout $t$.

Dans ces hypothèses (cf. [6] ou [7], p. 508-518) il existe un ensemble $K$ dans lequel le système (13) est relativement borné et, bien plus, pour toute solution $(x(t), y(t))$ de ce système il existe un $t_{0}$ tel que $\left(x\left(t_{0}\right), y\left(t_{0}\right)\right) \in K$. Pour tout point $(x, y) \in K$ on a les inégalités:

$$
|x| \leqslant x_{0}, \quad|y-F(x)| \leqslant y_{0},
$$

$x_{0}$ et $y_{0}$ étant deux constantes positives. Du théorème 3 on peut donc déduire le théorème suivant:

THÉORÈme 4. Si les fonctions $g(x), f(x)$ et $p(t)$ satisfont aus conditions (19)-(21) et aux inégalités

$$
f(x)>0, \quad y_{0}\left|g^{\prime \prime}(x)\right|<2 f(x) g^{\prime}(x) \quad\left(-x_{0} \leqslant x \leqslant x_{0}\right),
$$

pour deux solutions arbitraires $x_{1}(t), x_{2}(t)$ de l'équation (4) on a

$$
\lim _{t \rightarrow+\infty}\left|x_{1}(t)-x_{2}(t)\right|=0, \quad \lim _{t \rightarrow+\infty}\left|x_{1}^{\prime}(t)-x_{2}^{\prime}(t)\right|=0 .
$$

On obtient ainsi une généralisation d'un théorème de M. L. Oartwright et J. E. Littlewood (cf. [1], Théorème 2 et aussi [2], p. 271).

11. Oonsidérons le système d'équations différentielles

$$
x^{\prime}=y, \quad y^{\prime}=-F^{\prime}(y)-g(x)+p(t),
$$

équivalent à une seule équation différentielle du second ordre

$$
x^{\prime \prime}+F\left(x^{\prime}\right)+g(x)=p(t) .
$$

Pour le système (22) on peut démontrer un théorème analogue au théorème 3 (cf. aussi [4], théorème 4), ainsi qu'un corollaire analogue au corollaire 2.

THÉORÈme 5. Soit $K$ un ensemble plan dont la frontière est une courbe de classe $\tilde{O}^{1}$ et dans lequel le système (22) est relativement borné. Si les fonctions $g^{\prime \prime}(x), f(y)=F^{\prime}(y)$ et $p(t)$ sont continues et satisfont dans l'ensemble $K$ aux inégalités

$$
g^{\prime}(x)>0, \quad f(y) g^{\prime}(x)+g^{\prime \prime}(x) y>0,
$$

le système (22) est asymptotiquement stable dans l'ensemble $K$.

CoROLLAIRE 3. Si les hypothèses du théorème 5 sont vérifiées, toute solution $(x(t), y(t))$ du système (22) passant par un point intérieur de l'ensemble $K$ est asymptotiquement stable au sens de Liapounoff.

Pour la démonstration il suffit de remarquer que dans le cas envisagé le système (6) prend la forme suivante

$$
v_{1}^{\prime}=v_{2}, \quad v_{2}^{\prime}=-f(y(t)) v_{2}-g^{\prime}(x(t)) v_{1}
$$

et de répéter pour la fonction auxiliaire

$$
H(t)=v_{1}^{2}(t)+v_{2}^{2}(t) / g^{\prime}(x(t))
$$

tous les raisonnements de la démonstration du théorème 3 .

\section{Travaux cités}

[1] M. L. Cartwright and J. E. Littlewood, On non-linear differential equa tions of the second order II, Annals of Math. 48, No 2 (1947), p. 472-494.

[2] S. Lefschetz, Differential equations: geometric theory, New York 1957.

[3] И. Г. Малкин Теория устойчивости әвижения, Москва.Јенинград 1952.

[4] Z. Opial, Sur la stabilité des solutions périodiques et presque-périodiques de l'équetion différentielle $x^{\prime \prime}+F\left(x^{\prime}\right)+g(x)=p(t)$, Bull. Acad. Polon. Sci., Sér. des soi. math., phys et astr. 7 (1959), p. 493-498.

[5] - Sur les solutions périodiques et presque-périodiques de l'équation différentielle $x^{\prime \prime}+k f(x) x^{\prime}+g(x)=k p(t)$, ce volume, p. 309-319.

[6] G. E. H. Reuter, $A$ boundedness theorem for non-linear differential equations of the second order, Proc. Cambr. Phil. Soo. 47 (1951), p. $49-54$.

[7] G. Sansone, R. Conti, Equazioni differenziali non lineari, Roma 1956.

[8] G. Seifert, On stability in the large for periodio solution of differential systems, Annals of Math. 67, no 1 (1958), p. 83-89.

[9] - The asymptotic behavior of pendulum-type equations, Annals of Math. 69, No 1 (1959), p. 75-87.

Regu par la Rédaotion le 5. 5. 1959 